\title{
Massive Hemorrhage: The Role of Whole Blood Viscoelastic Assays
}

\author{
Emmanuelle Scala ${ }^{1}$ Carlo Marcucci ${ }^{1,2}$ \\ ${ }^{1}$ Department of Anesthesiology, Lausanne University Hospital, \\ Lausanne, Switzerland \\ ${ }^{2}$ Faculty of Biology and Medicine, University of Lausanne, Lausanne, \\ Switzerland
}

\author{
Address for correspondence Carlo Marcucci, MD, Department of \\ Anesthesiology, Centre Hospitalier Universitaire Vaudois (CHUV), \\ Rue du Bugnon 46, CH-1011 Lausanne, Switzerland \\ (e-mail: carlo.marcucci@chuv.ch).
}

\begin{abstract}
Keywords

- bleeding

- transfusion

- viscoelastic tests

Viscoelastic whole blood tests are increasingly used to guide hemostatic therapy in bleeding patients in the perioperative, trauma, and obstetric settings. Compared with standard laboratory tests of hemostasis, they have a shorter turnaround time and provide simultaneous information on various aspects of clot formation and lysis. The two available brands TEG (thromboelastography) and ROTEM (rotational thromboelastometry) provide devices that are either manually operated or fully automated. The automation allows for the assays to be used as point-of-care tests increasing their usefulness in massively bleeding patients with rapidly changing hemostatic profiles. While the number of research papers on the subject and the number of published treatment algorithms increase rapidly, the influence of the use of these devices on patient outcome needs yet to be established. In this article, we first review the technology of these devices and the parameters provided by the assays. Next, we present the problems encountered when choosing cut-off values that trigger intervention. Furthermore, we discuss the studies examining their influence on clinical outcomes, and finally, we briefly highlight some of the most important limitations and pitfalls inherent to these assays.
\end{abstract}

\section{Introduction}

Massive hemorrhage occurs in various clinical settings, most frequently in trauma, major surgery, and obstetrics, and is usually defined and graded by the quantity of blood products administered rather than by the amount of blood lost. Both processes, massive bleeding and massive transfusion, can lead to coagulopathic states, which complicate resuscitation efforts and compromise patient outcome. In short, the liberation of tissue factor into the systemic circulation leads to the activation of the coagulation, anticoagulation, and fibrinolysis pathways. ${ }^{1}$ The combined effects on hemostasis lead to early traumainduced coagulopathy (ETIC). ETIC is present in up to $25 \%$ of severely injured trauma patients ${ }^{2}$ and is characterized by excessive thrombin generation, platelet dysfunction, deficient fibrinogen, impaired fibrinolysis, and endothelial dysfunction, leading to a complex coagulopathy that can lead to both hypoand hypercoagulability. ${ }^{3}$ Circulating tissue factor can lead to a disseminated intravascular coagulation-like state with consumption of hemostatic elements on one hand and increased anticoagulation and fibrinolysis on the other hand. The latter pathways are enhanced due to the expression of thrombomodulin in poorly perfused tissues, which results in protein $C$ activation, the depletion of plasminogen activator inhibitor-1, and reduced activity of the thrombin-activatable fibrinolysis inhibitor. ${ }^{4}$ Massive volume resuscitation with crystalloids on the other hand leads to dilutional coagulopathy, anemia, and thrombocytopenia. The transfusion of large quantities of citrated blood products, in particular fresh frozen plasma (FFP), ${ }^{5}$ leads to calcium depletion. Acidosis and hypothermia received

May 6, 2020

accepted after revision

August 7, 2020 (c) 2020 Georg Thieme Verlag KG Stuttgart · New York
DOI https://doi.org/

$10.1055 / \mathrm{a}-1227-8050$ ISSN 0720-9355. 
further impair hemostasis. ${ }^{6}$ The past decade has seen a change in the practice of hemostatic resuscitation with the development of massive transfusion protocols (MTPs), which aim to restore hemodynamic stability, while optimizing hemostatic capacity. First reported by the U.S. army surgeons, ${ }^{7,8}$ the early administration of FFP in a high FFP to red blood cell (RBC) ratio improves patient survival. Soon after, platelet concentrates (PCs) have been added to the resuscitation protocols. The optimal ratio of FFP:PC:RBC products remains to be determined. Although a high ratio of 1:1:1 did not show a survival benefit compared with 1:1:2 in an randomized controlled trial (RCT) including a total of 680 severely injured trauma patients, the 1:1:1 ratio was associated with less death by exsanguination and better hemostasis. ${ }^{9}$ Today, most MTPs aim for the administration of RBC, FFP, and PC in a 1:1:1 ratio, combined with the administration of antifibrinolytic and procoagulant drugs, during the initial management of massive hemorrhage. ${ }^{10}$ Monitoring of acid-base status, oxygen carrying capacity, and hemostasis is an integral part of patient management but is particularly difficult for the latter. Standard blood cell counts and coagulation tests have a long turnaround time and do not provide useful information in patients who are transfused several units of various blood products before the result is known. The whole blood viscoelastic tests have gained popularity for the monitoring and guidance of hemostatic therapies during massive transfusion. In this short review, we will briefly present the technology and the derived parameters, and discuss the methods used for choosing the relevant parameters and their cut-off values in the setting of massive bleeding. Next, the clinical evidence supporting the use of viscoelastic tests will be presented. Finally, the limitations and pitfalls inherent to this technology will be explained.

\section{Whole Blood Viscoelastic Tests}

The two commercially available viscoelastic devices, thromboelastography (TEG) and rotational thromboelastometry (ROTEM), operate on the same basic principle: a pin is lowered into a cup, holding a sample of whole blood, after an activator of coagulation has been added. In TEG, the cup rotates around the pin in a to-and-fro movement describing a $4^{\circ} 45^{\prime}$ angle in 5 seconds. As the blood clots, and its viscosity increases, the pin is tethered and its increasing motion is detected and recorded by a torsion wire. In ROTEM, the pin rotates inside the cup making a $4^{\circ} 75^{\prime}$ angle in 6 seconds, and as the viscosity increases this motion is hampered and recorded contactless by a light-emitting diode light-mirror-light detector system. Both brands have commercialized fully automated devices, which can easily be used as pointof-care tests: TEG 6s and ROTEM sigma. The technology in the ROTEM sigma device is basically the same as in the semiautomated ROTEM delta device. TEG 6s however uses the measurement of resonance frequency to measure viscoelasticity. All devices provide a graphical display of the changes in viscoelasticity over time. The resulting graph provides information on coagulation factors, thrombin generation, and on the fibrinogen-platelet interaction, within minutes. -Fig. 1 shows the shape of a typical graph

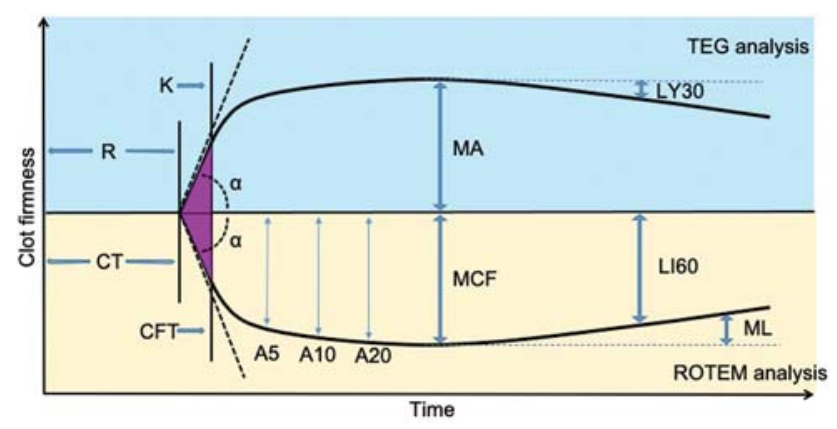

Fig. 1 Viscoelastic test curve and parameters. The Lag time from the onset of the test to the start of increasing viscoelasticity is represented by CT in ROTEM (expressed in seconds) and by R-time in TEG (expressed in minutes). The time from the start of the slope to an amplitude of $20 \mathrm{~mm}$ is the CFT in ROTEM (s) and K-time in TEG (min). Alpha is the angle between the zero line and the tangent through the point of $2 \mathrm{~mm}$ amplitude. A5, A10, and A20 are amplitudes measured at 5, 10, and 20 minutes after $C T$ and are expressed in millimeters. The maximum amplitude measured is called MCF in ROTEM and MA in TEG and is expressed in millimeters. LY and ML are indexes of thrombolysis expressed in percentages of loss of clot strength and $\mathrm{LI}$ as percentage residual clot strength. CFT, clot formation time; $C T$, coagulation time; LI60, lysis index at 60 minutes after CT; LY30, lysis at 30 minutes after MA; MA, maximum amplitude; MCF, maximum clot firmness; ML, maximum lysis index during runtime; TEG, thromboelastography. (Modified with permission from Heim E, Schoettker P. Viscoelastic tests of hemostasis. In: Marcucci C, Schoettker P, eds. Perioperative Hemostasis. 1st ed. Heidelberg: Springer; 2015:25-43.)

and the derived parameters. TEG and ROTEM measure similar parameters but use different terminologies.

After a lag time, during which the activator initiates either the intrinsic or the extrinsic pathways, the thrombin generated will cleave fibrinogen, activate factor XIII, and activate platelets. Subsequent clot formation will be apparent by a symmetric up- and down-slope of the curve, the slope of which corresponds to the velocity of thrombin generation and platelet-fibrin interaction. The height of the curve, expressed in millimeters, represents the strength of the clot, and is measured at various time points, until the maximum strength is reached. Thrombin will also initiate the fibrinolytic pathway, responsible for the loss in clot strength after the maximum amplitude has been reached. Lysis is quantified as percentage loss in viscoelasticity compared with the maximum value measured.

The duration of the lag time is dependent on coagulation factor activity and is named R-time or activated clotting time (ACT) in TEG and coagulation time (CT) in ROTEM. The rate of clot formation is dependent on thrombin generation, fibrinogen concentration, and platelet count and activity. The rate of clot formation is quantified by two parameters: (1) the angle $\alpha$, which is measured between the baseline and the tangent of the slope at $2 \mathrm{~mm}$ of amplitude and (2) the time for the slope to reach an amplitude of $20 \mathrm{~mm}$ : $K$-time (TEG) and clot formation time (ROTEM). The amplitude of the curve depends on the concentration of fibrinogen, platelets, and factor XIII activity. The maximum amplitude reached is termed maximum amplitude (MA) in TEG and maximal clot firmness (MCF) in ROTEM. In ROTEM, amplitudes are measured at various time points $\left(A_{n}\right.$ : amplitude at $n$ minutes after $\mathrm{CT}$ ). Early amplitudes are not 
validated in TEG, resulting in a delay in obtaining results compared with ROTEM. Finally, lysis is measured at various time points and is defined differently regarding timing. In both devices, lysis is represented by maximum lysis, which represents the maximum percent loss of clot strength compared with MCF during the runtime. In ROTEM, the lysis index (LI) expresses the percentage of residual clot strength. LI30,45, and 60 are detected 30,45 , and 60 minutes after CT, respectively. In TEG, the $L Y$ parameter is the calculated percent loss of clot strength compared with MA. LY30 and LY60 are detected at 30 and 60 minutes after MA, respectively. Since time to MA takes a mean of 25 minutes for rapid-TEG and 43 minutes for kaolinTEG, ROTEM LI60 is measured at a similar runtime to TEG LY30. Additional ROTEM fibrinolysis parameters are lysis onset time (in seconds) and lysis time (in seconds) defined as the time from CT until clot firmness is decreased by $15 \%$ compared with MCF and the time from CT until clot firmness is decreased to $10 \%$ of MCF, respectively.

\section{Assays and Time-Dependent Parameters}

\section{Assays}

In viscoelastic tests, coagulation is initiated through the extrinsic and/or intrinsic pathways. The addition of specific reagents allows for better discrimination between different coagulopathies ( - Table 1 ). The contribution of fibrin to the total clot strength can be evaluated by adding a platelet inhibitor. Polybrene (EXTEM, FIBTEM, and APTEM) or heparinase (HEPTEM, NaHEPTEM, and kaolin-TEG plus heparinase), which inactivate heparin, are added to exclude or identify a heparin effect on hemostasis. The addition of an antifibrinolytic drug (aprotinin or tranexamic acid) can simulate the effect of an antifibrinolytic drug in vitro (specific for ROTEM).

TEG offers integrated platelet function testing by activating the blood sample with reptilase, creating a fibrin mesh upon which platelets aggregate after adding specific platelet activators (arachidonic acid, adenosine diphosphate, or thrombin receptor-activating peptide-6). The ROTEM delta device offers platelet function testing (ROTEM platelet), using whole blood impedance aggregometry and the activators arachidonic acid (ARATEM), adenosine diphosphate (ADPTEM), and thrombin receptor-activating peptide-6 (TRAPTEM).

\section{Time-Dependent Parameters}

When treating bleeding patients, time is of the essence. Although viscoelastic tests have a relatively short turnaround time, depending on the assay used, the first results can be read within minutes from the start of the test or can take up to 30 minutes to become available. Moreover, some of the parameters such as MAs and LIs only appear, by definition, after 30 minutes or more. Specifically, in the classic native-TEG assay, which uses no activator, the lag time can take up to 30 minutes. Adding activators of the intrinsic and/or extrinsic pathways will shorten the time to results, but may theoretically mask underlying deficiencies. Coleman et al ${ }^{11}$ retrospectively compared native-TEG, kaolin-TEG, and rapid-TEG in their capability of predicting massive transfusion or death in a population of 343 trauma patients. The area under the receiver operating characteristic (ROC) curve for R-time was 0.5984 (95\% confidence interval [CI]: 0.4995-0.6973) for rapid-TEG, 0.6431 (95\% CI: 0.4995-0.6973) for native-TEG, and 0.7033 (95\% CI: 0.6131-0.7935) for kaolin-TEG. The areas under the ROC curve (AUCs) for MA were 0.7955 (95\% CI: 0.7108-0.8802) for rapid-TEG, 08268 (95\% CI: 0.7468-0.9068) for native-TEG, and 0.7984 (95\% CI: 0.7133-0.8835) for kaolin-TEG. Although, rapid-TEG performed less well for R-time and slightly less for MA, the 95\% CIs showed great overlap. The authors concluded that overall, the rapid-TEG's performance was noninferior to the two other assays and can be used to gain time in the diagnosis and treatment of ETIC.

Several authors have investigated the relationship between early amplitudes (A5, A10) and MAs, and consistently found very strong correlations, indicating that early amplitudes predict MAs with high reliability, allowing algorithms to use

Table 1 Viscoelastic test assays, activators, and adjuncts for ROTEM and TEG

\begin{tabular}{|c|c|c|c|c|}
\hline Brand & Test name & Activator & Adjunct & Test goal \\
\hline \multirow[t]{8}{*}{ ROTEM } & EXTEM & Tissue factor & & Extrinsic pathway activation \\
\hline & INTEM & Ellagic acid & & Intrinsic pathway activation \\
\hline & FIBTEM & Tissue factor & Cytochalasin D & Fibrin contribution \\
\hline & HEPTEM & Ellagic acid & Heparinase & Heparin presence \\
\hline & APTEM & Tissue factor & Aprotinin & Hyperfibrinolysis \\
\hline & tAPTEM & Tissue factor & Tranexamic acid & Hyperfibrinolysis \\
\hline & NATEM & None & & Tissue factor presence \\
\hline & NaHEPTEM & None & Heparinase & LMWH presence \\
\hline \multirow[t]{5}{*}{ TEG } & Standard TEG (KaoTEG) & Kaolin & & Intrinsic pathway activation \\
\hline & Rapid TEG (r-TEG) & Tissue factor and kaolin & & Mixed pathway activation \\
\hline & HEPARINASE (HTEG) & Tissue factor or kaolin & Heparinase & Heparin presence \\
\hline & Functional fbg (FF) & Tissue factor & Abciximab & Fibrin contribution to clot \\
\hline & Native TEG & None & & Spontaneous clot formation \\
\hline
\end{tabular}

Abbreviations: LMWH, low-molecular-weight heparin; ROTEM, rotational thromboelastometry; TEG, thromboelastography. 
A5 or A10 to trigger therapeutic interventions. In a retrospective analysis of ROTEM tests performed in 1,146 trauma patients on admission, the A5 and A10 amplitudes of the EXTEM assay, respectively, showed Pearson correlation coefficients of 0.92 and 0.96 with MCF. More importantly, the ROCs of A5 and A10 in predicting mortality or massive transfusion were identical to those of MCF. ${ }^{12} \mathrm{~A}$ prospective analysis in 404 trauma patients of TEG parameters found similar results. ${ }^{13} \mathrm{The}$ Spearman correlation was very strong for the early amplitudes A5 and A10 with MA in the rapid-TEG assay (coefficients of 0.86 and 0.90 ), the functional fibrinogen test (0.87 and 0.90 ), and just slightly less strong for the kaolin-TEG assay ( 0.77 and 0.89 ). Decreased amplitudes at all time points were highly significantly associated with increased transfusion requirements. A logistic regression analysis in transfused patients showed that reduced early amplitudes $\mathrm{A} 5$ and $\mathrm{A} 10$ in the rapidTEG and kaolin-TEG assays were significant risk factors for mortality, where the MAs were not. In the functional fibrinogen test, low A5, A10, and MA were associated with increased mortality. The authors also reported the time to result from the beginning of the tests, which were 6 minutes for A5 in the rapid-TEG and functional fibrinogen tests and 10 minutes for A5 in the kaolin test, compared with 18, 16, and 22 minutes, respectively, for MA.

Specifically for the ROTEM sigma model, Scala et al found very strong correlations of A5 and MCF in the EXTEM $(r=0.96)$, FIBTEM $(r=0.99)$, and the INTEM $(r=0.98)$ tests. ${ }^{14}$ Based on these results, the authors recommend the use of A5 parameters for all tests in ROTEM-based transfusion algorithms.

Early clot firmness parameters have not been approved for TEG, yet. Therefore, at this time, these parameters cannot be used in TEG-based algorithms.

\section{Cut-Off Values}

It is important to realize that the parameters of viscoelastic tests depend on the concentration of hemostatic elements, their function, and their interaction. The results therefore do not quantify the individual hemostatic elements contrary to standard laboratory tests (SLT) such as prothrombin time, activated partial thromboplastin time (aPTT), fibrinogen concentration, or platelet count. The known ranges of normal values of the viscoelastic tests represent nothing more than the 2.5th and 97.5th percentiles of values found in healthy populations. They do not represent the lower ranges of SLTs, nor do they reflect cut-off values of SLTs generally recommended as treatment triggers. ${ }^{15}$ Also, although based on the same principles, the differences in reagents, their concentrations, and different measurement technologies between the two brands and between devices of the same brand imply that the results are not interchangeable. ${ }^{16}$ To establish treatment algorithms, device specific cut-off values have to be determined. This can be done by ROC analyses determining the values that predict either SLT triggers or clinical outcomes with the best specificity and sensitivity. Bouzat et al ${ }^{17}$ investigated both ROTEM delta and sigma models and found similar sensitivities and specificities for identical cutoff values, and concluded that trauma centers can switch between these two models without adapting. An A5 of $<7 \mathrm{~mm}$ in the FIBTEM predicted a fibrinogen concentration of $<1.5 \mathrm{~g} / \mathrm{L}$ with a sensitivity of $83 \%$ and a specificity of $82 \%$ on the ROTEM sigma and 85 and $90 \%$, respectively, on the ROTEM delta model. In contrast, the algorithm developed in our center ${ }^{14}$ ( - Fig. 2) uses a combination of A5 of $<12 \mathrm{~mm}$ and in the FIBTEM as a treatment trigger, since in our analysis this cut-off predicted $<1.5 \mathrm{~g} / \mathrm{L}$ of fibrinogen with $100 \%$ sensitivity and $64 \%$ specificity. This illustrates that defining cut-offs depends not only on the make and model of the device, but also on the population tested, the relative importance attributed to sensitivity and specificity of each value, and the rationale behind the construction of the algorithm.

Since the cut-off values of SLTs have been shown to have low positive and negative predictive values for bleeding, the viscoelastic parameters predicting these values will likely have the same limitations. Ideally, trigger values for treatment are based on clinical outcomes. ${ }^{18,19}$

Stettler et al used the clinical outcome parameter of massive transfusion, defined as $>10$ units of RBC in 6 hours after admission, or death within the same time period to identify treatment triggers with the best AUC for both kaolin-TEG $(n=825$ patients $)$ and ROTEM $\left(n=222\right.$ patients). ${ }^{20,21}$ The models used were the TEG 5000 thromboelastograph hemostasis analyzer and the ROTEM delta. The TEG triggers for different therapeutic interventions proposed are an R-time $>4.45$ minutes for FFP transfusion, $\alpha$ angle $<67^{\circ}$ for the use of fibrinogen products, an $\mathrm{MA}<67 \mathrm{~mm}$ for PC administration, and $\mathrm{LY} 30>4.55 \%$ for antifibrinolytics. Although in their study evaluating ROTEM three different tests were used (EXTEM, FIBTEM, and APTEM), all the parameters they propose for treatment are derived from the EXTEM assay, since these yielded, in their population, the best balance between sensitivity and specificity: EXTEM CT $>78.5$ seconds for plasma transfusion, $\alpha$ angle $<64.5^{\circ}$ for fibrinogen products, EXTEM A $10<40.5 \mathrm{~mm}$ for PC administration, and EXTEM LI60 $<74 \%$ for antifibrinolytic therapy. Interestingly, using the APTEM test and comparing the CT with the EXTEM CT unveil fibrinolysis in a matter of minutes with better specificity and negative predictive values than LI60. Nevertheless, the authors did not retain this method as an early indication for antifibrinolytic therapy since its sensitivity and positive predictive value were much lower than the LI60 parameters. This again illustrates that published cut-offs are inevitably the result of a trade-off, and algorithms will vary according to balanced choices, institutional standards, and regional practices.

\section{Viscoelastic Tests and Outcomes in Massive Transfusion}

Since the basis of many MTPs is the immediate and indiscriminate transfusion of RBC, FFP, and PC in fixed ratios, the role of hemostatic testing can be questioned and is hard to establish. Very few RCTs addressing this issue have been performed and a definitive answer cannot yet be given. The most recent meta-analysis evaluating viscoelastic assays in the perioperative period includes cardiac surgery (16 studies), liver surgery (2 studies), orthopedic surgery (2 studies), 


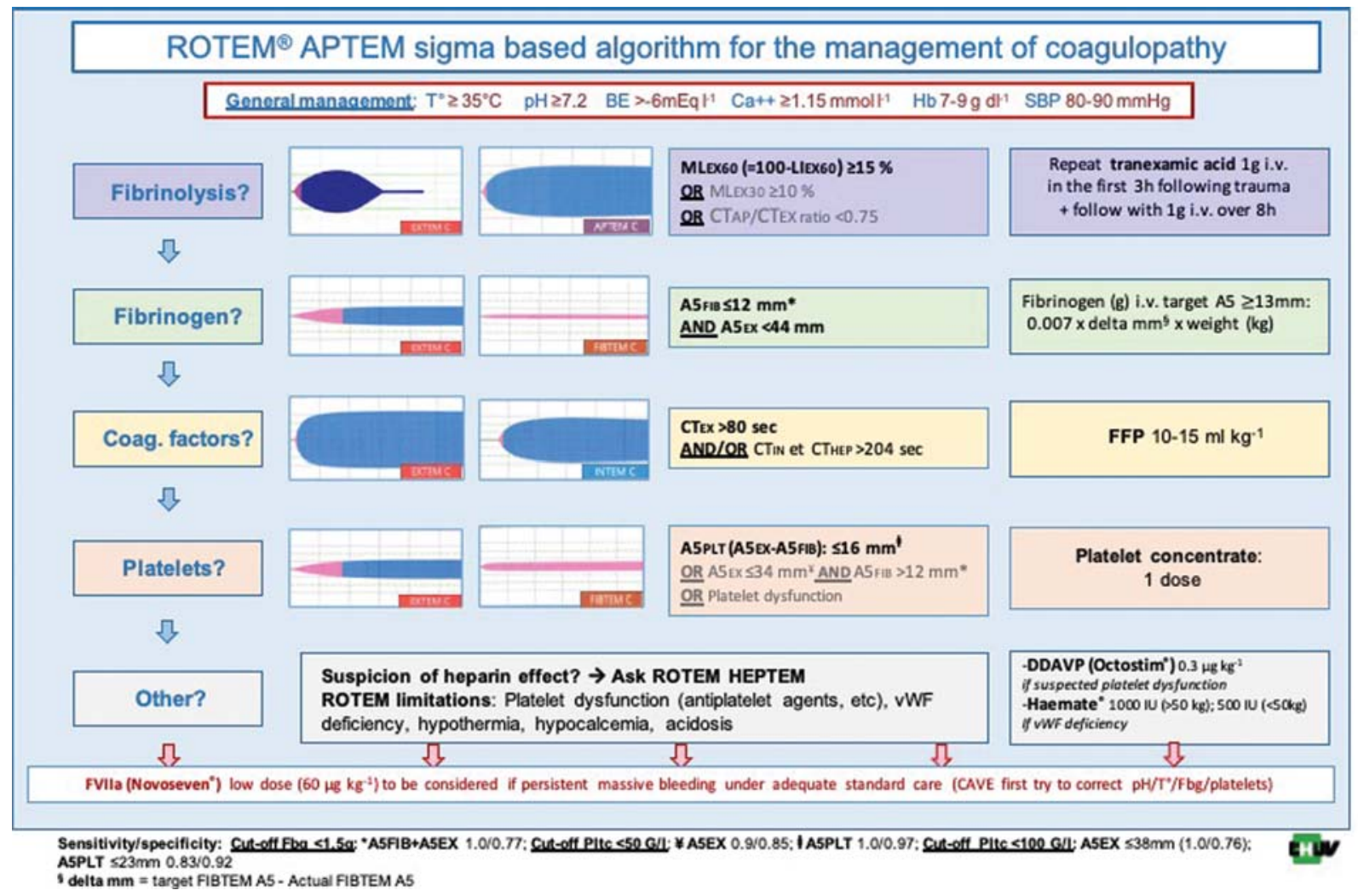

Fig. 2 Stepwise treatment algorithm based on ROTEM test results for ROTEM sigma. Specificities and sensitivities of the cut-off values are marked as a footnote. $A 5$, amplitude at 5 minutes after $C T$; $A 5_{P L T}$, platelet contribution to clot firmness calculated as $A 5_{E X}-A 5_{F I B}$; $A P, A P T E M$ test; $\mathrm{BE}$, base excess; Ca, calcium; CT, coagulation time; EX, EXTEM test; Fbg, plasma fibrinogen level measured by the Clauss method; FFP, fresh frozen plasma; FIB, FIBTEM test; Hb, hemoglobin; i.v. , intravenous; LI60 is defined as the residual clot firmness in percentage of MCF at 60 minutes after $C T ; \mathrm{ML}_{\mathrm{EX} 60}$, maximum lysis at 60 minutes in the EXTEM test; Pltc, platelet count; ROTEM, rotational thromboelastometry; SBP, systolic blood pressure; vWF, von Willebrand factor. (Reproduced with permission form Scala E, Coutaz C, Gomez F, Alberio L, Marcucci C. Comparison of ROTEM sigma to standard laboratory tests and development of an algorithm for the management of coagulopathic bleeding in a tertiary center. J Cardiothorac Vasc Anesth 2020;34(3):640-649.)

trauma ( 1 study), and burn victims ( 1 study). ${ }^{22}$ Compared with standard care (defined as decision based on SLTs and/or clinical judgment), the use of viscoelastic assays resulted in a reduction of the risk of death (risk ratio $[\mathrm{RR}]=0.65 ; 95 \% \mathrm{CI}$ : $0.43-0.96)$ and reduced kidney injury $(\mathrm{RR}=0.53 ; 95 \% \mathrm{CI}$ : 0.34-0.83). The lower risk of RBC transfusion in the viscoelastic assay groups did not reach statistical significance, nor did the rates of reoperation for bleeding.

\section{Cardiac Surgery}

Specifically in cardiac surgery, 2016 Cochrane meta-analysis $^{23}$ identified 17 RCTs, involving 1,493 participants, comparing the use of viscoelastic-test-based algorithms versus any other treatment. Fifteen of these trials were performed in cardiac surgery, one in liver transplant surgery, and one during excision of burn wounds. Overall, they found that treatment algorithms based on either TEG or ROTEM reduced the patients' risk of mortality (RR: 0.52; 95\% CI: 0.28-0.95), RBC transfusion (RR: 0.86; 95\% CI: 0.79-0.94), FFP transfusion (RR: 0.57; 95\% CI: 0.33-0.96), PC transfusion (RR: 0.73; 95\% CI: 0.60-0.88), and dialysis-dependent renal failure (RR: 0.46; 95\% CI: 0.28-0.76). A more recent meta-analysis compiled data of 15,320 participants exclusively in adult cardiac surgery. ${ }^{24}$ The much higher number of patients included come mostly from several large retrospective cohort studies $^{25-27}$ and one major RCT. ${ }^{28}$ They found similar results on the risk reduction of the use of blood products: $0.89(95 \% \mathrm{CI}$ : $0.80-0.98$ ) for RBC transfusion, 0.59 (95\% CI: $0.42-0.82$ ) for FFP transfusion, and 0.81 (95\% CI: 0.74-0.90) for PC transfusion, and also found an overall reduction of blood loss volume by $132 \mathrm{~mL}$ (95\% CI: 207-57). In spite of these encouraging results, the authors call for "great caution" in interpreting them because of the imprecision, inadequate power, and significant bias in most of the trials.

Also, elective cardiac surgery can result in severe bleeding, but cannot be compared with the uncontrolled and unpredictable settings of trauma or postpartum hemorrhage.

\section{Trauma}

At this time, only a few trials on the influence of viscoelastic testing on clinical outcomes in trauma patients are available. Prat et $\mathrm{al}^{29}$ compared transfusion practices and mortality in damage control resuscitation on combat casualties before and after the introduction of ROTEM testing in the Bagram Airfield Military Hospital. Blood samples from victims were tested on a ROTEM delta device as of November 2011; the results were available for the physicians but no specific protocol was used. A total of 134 pre-ROTEM patients were 
compared with 85 post-ROTEM. No differences in clinical outcome or mortality were observed. In the ROTEM era, significantly more platelets and cryoprecipitate were transfused, showing that the use of a viscoelastic test may lead to a more patient-centered hemostatic management. A recent retrospective analysis examined the role of the use of TEG during initial resuscitation in civilian trauma. Unruh et al compared outcomes and blood product use in trauma patients managed with $(n=47)$ or without TEG guidance $(n=20)$ during the activation of a MTP. ${ }^{30}$ The protocol aims for a 1:1:1 ratio of blood products but allows for deviation at the physician's discretion. The TEG-based guidelines are as follows: R-time $>10$ seconds: transfuse 2 FFPs; Ktime $>3$ seconds: transfuse 1 unit of cryoprecipitate; alpha angle $<53^{\circ}$ : transfuse 1 unit of cryoprecipitate; $\mathrm{MA}<50 \mathrm{~mm}$ : transfuse 1 unit of PC; LY30 > 3\%: administer tranexamic acid. The baseline hematocrit was significantly higher in the TEG-treated group (34.5 vs. 29.7, $p<0.022$ ), which may partially account for the lower rate of RBCs transfused (6 vs. 11 units, $p<0.001$ ). But with similar demographic characteristics (type and severity of injury), similar clinical signs of hypovolemia and similar baseline values for the standard coagulation tests (aPTT, international normalized ratio [INR], and fibrinogen), the use of FFP and PC was significantly lower in the TEG group than in the non-TEG group. A total of $17 \%$ of patients in the TEG group received FFP compared with $85 \%$ in non-TG-treated patients, and $38 \%$ of TEG patients received PC versus $75 \%$ in non-TEG-patients. Although mortality tended to be lower in the TEG group ( 31.9 vs. $55.0 \%$, the difference did not reach statistical significance. As for intensive care length of stay, duration of mechanical ventilation, and hospital length of stay, no difference between the groups was observed.

Stein et $\mathrm{al}^{31}$ evaluated the impact of the implementation of a ROTEM-guided treatment algorithm in trauma care on the use of blood products and hemostatic agents. They retrospectively compared 323 patients treated before (2005-2007) the introduction of the algorithm with 408 patients treated after (2012-2014) its implementation. They found a reduction of massive transfusion from 12 to $4 \%(p<0.001)$, FFP transfusion in the emergency room from 31 to $6 \%(p<0.001)$, and overall mortality from 33 to $22 \%(p<0.001)$. The use of prothrombin complex concentrate and factor XIII concentrate, on the other hand, increased the massive transfusion. This study is limited by its retrospective design comparing patients from two different epochs. It is important to note that other changes in the management strategy may have influenced the results, notably the routine use of tranexamic acid and the change of colloid solution used for volume resuscitation after the introduction of the algorithm.

In the only RCT to date, TEG (TEG-5000) was compared with standard coagulation assays upon MTP activation in trauma. ${ }^{32}$ Out of a total of 111 patients, 56 were randomized to the TEG arm and 55 to the standard assay arm. During patient management the physicians in charge demanded unblinding of the TEG results in eight patients of the standard assay arm. The criteria for the administration of blood products in the TEG arm are significantly different from the one used in the above-mentioned study by Unruh et al. $^{30} \mathrm{~A}$ TEG-ACT value of $>140$ seconds prompted the transfusion of two FFPs, one unit of cryoprecipitate, and one unit of PC before the other parameters were available. Otherwise, for an ACT between 111 and 139 seconds, two FFPs were transfused, cryoprecipitate for an angle $<63^{\circ}$, and $\mathrm{PC}$ for a $\mathrm{MA}<55 \mathrm{~mm}$. In the standard assay arm, thresholds for FFP, cryoprecipitate, and platelet transfusion were an INR $>1.5$, fibrinogen $<1.5 \mathrm{~g} / \mathrm{L}$, and platelet count $<100 \mathrm{G} / \mathrm{L}$.

The 28-day survival was significantly better in the TEGtreated group in the intention to treat analysis. Mortality was $19.6 \%$ in the TEG group and $36.4 \%(p=0.049)$ in the standard assay group. The difference became even more important in the as-treated analysis: 18.7 versus $40.4 \%(p=0.011)$. While there was no difference in RBC transfusion in the first 24 hours after admission, the rates of FFP and PC transfusion were significantly lower in the TEG group during the first 2 to 4 hours of resuscitation and the rate of cryoprecipitate transfusion was lower in the TEG group during the first 24 hours after admission. The authors argue that although the total volume of blood products administered did not differ between the two groups, the optimized timing of component transfusion explains the survival benefit in the TEG group.

\section{Obstetrics}

In obstetrics Snegovskikh et al report on a retrospective cohort analysis comparing the outcomes of postpartum hemorrhage between two historical groups of parturients. ${ }^{33}$ The first group of 58 patients was treated according to a standard MTP before the introduction of ROTEM, and the second group of 28 patients was treated according to a ROTEM-based algorithm (ROTEM-delta). Postpartum hemorrhage was defined as an estimated blood loss of $1,500 \mathrm{~mL}$ or more. Median estimated blood loss in the ROTEM group was $2,000 \mathrm{~mL}(1,600-2,500)$, and significantly lower than the $3,000 \mathrm{~mL}(2,000-4,000)$ in the standard group $(p<0.001)$. Also, the amounts of RBCs, FFP, and PC transfused were much lower in the ROTEM group ( $p<0.0001$ for all comparisons), as were intensive care unit (ICU) admission, hospital length of stay, and, importantly, the rate of puerperal hysterectomy.

Finally, Mcnamara et $\mathrm{al}^{34}$ retrospectively analyzed the data from ROTEM delta tests in parturients showing major obstetric hemorrhage. They compared the outcomes of 52 woman treated with a standardized shock pack with the outcomes of 203 woman treated according to a ROTEM-delta-guided algorithm. In the ROTEM-guided algorithm, fibrinogen concentrate was administered if the FIBTEM A5 was less than $7 \mathrm{~mm}$, or if it was between 7 and $12 \mathrm{~mm}$ with ongoing bleeding, and FFPs were administered if the EXTEM CT was higher than 100 seconds. They found that the ROTEM-guided management resulted in a strong reduction in the use of blood products and a significant decrease in the incidence of transfusion-associated circulatory overload. ICU admission and the incidence of hysterectomy both tended to be lower in the ROTEM-guided treatment group without reaching statistical significance. When analyzing the association of the different ROTEM parameters with severity of bleeding, they found that an EXTEM CT of $>100$ seconds was predictive of low FIBTEM 
and EXTEM amplitudes and indicated severe coagulopathy. This degree of severe coagulopathic bleeding was most associated to placental abruption and intrauterine death. On the other hand, FIBTEM A5 in itself only showed a weak negative correlation with the amount of blood loss and the total dose of fibrinogen administered.

\section{Limitations and Pitfalls}

The biggest advantage of point-of-care tests, i.e., the almost immediate availability of the results, is also their biggest disadvantage. SLTs are performed by trained medical laboratory technicians and validated by specialized medical staff. Pointof-care tests are often performed by physicians in charge of clinical care after minimal training. Especially in the manually operated devices, this can easily lead to artifacts in the viscoelasticity curves and erroneous results that need to be recognized. Also, various frequent pathological conditions are not detected by the viscoelastic tests. A multicenter trial evaluated adherence to a ROTEM-guided treatment algorithm in patients with traumatic brain injury. ${ }^{35}$ Participating physicians received a 2-day training in operating with a ROTEM sigma device, interpreting the results, and applying them to the treatment algorithm. Overall, the algorithm was followed with complete adherence in 20 out of 32 patients. For the individual steps of the algorithm, adherence was 88 to $91 \%$. Nevertheless, in five out of 32 cases, the treatment algorithm was not followed due to misinterpretation of the results. Without validation of the results by hematologists, physicians have to be familiar with these assays and their limitations to avoid treating artifacts and to avoid the unrecognition of underlying pathologies or conditions that are not identified by these assays. The aim of this section is to give the clinicians a quick look at the most frequent pitfalls that have to be known to adequately treat the coagulopathy associated with major bleeding.

\section{Patient-Related Factors}

The most relevant limitation in our industrialized countries is the inability to identify antiaggregant effect. When coagulation is initiated in viscoelastic tests, thrombin is generated in supraphysiologic concentrations and activates the platelet via its thrombin receptor even in the presence of inhibitors of the P2Y12 or thromboxane pathways. ${ }^{36}$ Several point-ofcare platelet function tests are available and can even, in the case of the TEG $6 \mathrm{~s}$, be run on the same device. Concomitant use with viscoelastic tests is likely to increase diagnostic accuracy and can help to optimize therapy. A detailed discussion of these tests is beyond the scope of this article and we encourage the reader to explore the growing body of literature on the subject.

Also, Von Willebrand disease (VWD) is the most common congenital coagulopathy ${ }^{37}$ and must be recognized in the context of acute bleeding, particularly if the patient is unknown in the treating hospital or has never been diagnosed. The principles used in ROTEM or TEG do not include the high shear stress as it occurs in endothelial lesions. This implies that VWD will not be recognized, unless there is an associated low factor VIII, ${ }^{36}$ as it is the case in type IIN VWD. ${ }^{38}$
Another limitation is the fact that these tests do not reflect in vivo hemostasis, particularly, the function of vascular endothelium, including the antithrombotic function of cell-bound thrombomodulin. ${ }^{36}$

\section{Device-Related Factors}

One must keep in mind the well-known high coefficient of variation, particularly for TEG, ${ }^{39}$ even if the within-device repeatability seems to be better for the newer model TEG $6 \mathrm{~s} .{ }^{40}$

In both TEG and ROTEM, the blood samples are heated to a predefined temperature, which is usually set at $37^{\circ} \mathrm{C}$, so that any coagulopathy associated with hypothermia will not be detected in the in vitro coagulation test. The test temperature can be modified, and the effect of temperature on the parameters of both brands have been studied. ${ }^{41,42}$ The clinical applicability and the consequences on interpretation and therapeutic interventions remain unknown.

The reagents contain calcium to recalcify the blood sampled in citrated tubes, and this over-concentration of calcium will mask any underlying hypocalcemia in the patients; it is therefore mandatory to monitor calcium levels using blood gas analysis.

Last but not the least, EXTEM, FIBTEM, and APTEM contain Polybrene for neutralization of up to $5 \mathrm{IU} / \mathrm{mL}$ of heparin in both ROTEM delta and ROTEM sigma devices. This is done to avoid any interference with either exogenous heparin or heparinlike molecules expressed, for example, during sepsis/systemic inflammatory response syndrome ${ }^{43}$ and acute liver failure during liver disease ${ }^{44}$ or during extracorporeal membrane oxygenation following cardiotomy. ${ }^{45}$ The INTEM/HEPTEMCT ratio can be used to detect unfractionated heparin and correlates well with anti-Xa activity. ${ }^{46}$ The NATEM/NaHEPTEM-CT ratio can be used to detect low-molecular-weight heparin. Notably, rapid-TEG and TEG-FF in TEG 6s do not contain a heparin inhibitor. Therefore, results of these tests cannot be used for decision-making if blood samples containing heparin were used. At this time kaolin-TEG with heparinase is the only assay included in the TEG 6s cartridge, which can be interpreted adequately. Accordingly, TEG 6 s analysis is recommended after heparin reversal in cardiac surgery, which can result in a delay in complex cardiac surgery.

\section{Future Developments}

New parameters on both ROTEM ${ }^{47}$ and TEG $^{48}$ are becoming available on the devices. One of these is the first derivative of the viscoelastic curve, which yields data on the maximum thrombin generation rate, area under the thrombin generation curve, and time to maximum thrombin generation. The clinical usefulness of these parameters has not been established yet.

The classic pin and cup technology dates back to the late1940s. Although it has undergone substantial technological changes in both automation and informatization in the ROTEM delta and sigma models and in the TEG 5000 model, the underlying principle has remained the same.

TEG has moved away from this technology in the TEG 6s model, which is based on the observation that the resonant frequency of blood is proportional to its viscosity. In the past 
5 to 6 years, several other devices based on other characteristics of clotting blood have been developed and some have been commercialized. These include sonic estimation of elasticity via resonance (Quantra Hemostasis Analyzer; HemoSonics, Charlottesville, Virginia, United States), ${ }^{49}$ laser speckle rheometry, ${ }^{50}$ mechanical resonant frequency (Abram Scientific Inc., Menlo Park, California, United States), ultrasonic deformation (Levisonics Inc., New Orleans, Louisiana, United States), and parallel plate viscometry (Viscoelastic Coagulation Monitor [VCM]; Entegrion Inc., Durham, North Carolina, United States). There is little to no experience with these devices, and their use in patient management needs yet to be established. For further information on the technical aspects of these devices and technologies, we recommend the comprehensive review article written by Hartmann et al. ${ }^{51}$

\section{Conclusion}

Viscoelastic tests are increasingly used to guide hemostatic therapy in patients with severe bleeding. Algorithms based on these assays show important differences in cut-off values which should be determined for the various devices and specific clinical settings and populations, in adequately powered prospective observational trials. Clinicians using viscoelastic tests as point-of-care tests for patient management must be familiar with the underlying technology and related limitations. The obvious advantage of goal-directed therapy and gain in time to treatment have yet to show improvement in patient outcome. A few trials in massively bleeding patients point in that direction but more high-grade evidence is needed.

\section{Disclosures}

The authors received logistical support from Axon Lab, Switzerland, for research activities.

\section{Conflicts of Interest}

Dr. Marcucci reports nonfinancial support from Axon Lab, during the conduct of the study. Dr. Scala reports nonfinancial support from Axon Lab, during the conduct of the study.

\section{References}

1 Pham HP, Shaz BH. Update on massive transfusion. Br J Anaesth 2013;111(Suppl 1):i71-i82

2 Fröhlich M, Mutschler M, Caspers M, et al; TraumaRegister DGU. Trauma-induced coagulopathy upon emergency room arrival: still a significant problem despite increased awareness and management? Eur J Trauma Emerg Surg 2019;45(01):115-124

3 Moore HB, Gando S, Iba T, et al; Subcommittees on Fibrinolysis, Disseminated Intravascular Coagulation, and Perioperative and Critical Care Thrombosis and Hemostasis. Defining trauma-induced coagulopathy with respect to future implications for patient management: communication from the SSC of the ISTH. J Thromb Haemost 2020;18(03):740-747

4 Brohi K, Cohen MJ, Davenport RA. Acute coagulopathy of trauma: mechanism, identification and effect. Curr Opin Crit Care 2007;13 (06):680-685

5 Moore HB, Tessmer MT, Moore EE, et al. Forgot calcium? Admission ionized-calcium in two civilian randomized controlled trials of prehospital plasma for traumatic hemorrhagic shock. J Trauma Acute Care Surg 2020;88(05):588-596

6 Spahn DR, Rossaint R. Coagulopathy and blood component transfusion in trauma. Br J Anaesth 2005;95(02):130-139

7 Borgman MA, Spinella PC, Perkins JG, et al. The ratio of blood products transfused affects mortality in patients receiving massive transfusions at a combat support hospital. J Trauma 2007;63 (04):805-813

8 Hess JR, Holcomb JB, Hoyt DB. Damage control resuscitation: the need for specific blood products to treat the coagulopathy of trauma. Transfusion 2006;46(05):685-686

9 Holcomb JB, Tilley BC, Baraniuk S, et al; PROPPR Study Group. Transfusion of plasma, platelets, and red blood cells in a 1:1:1 vs a $1: 1: 2$ ratio and mortality in patients with severe trauma: the PROPPR randomized clinical trial. JAMA 2015;313(05):471-482

10 Spahn DR, Bouillon B, Cerny V, et al. The European guideline on management of major bleeding and coagulopathy following trauma: fifth edition. Crit Care 2019;23(01):98

11 Coleman JR, Moore EE, Chapman MP, et al. Rapid TEG efficiently guides hemostatic resuscitation in trauma patients. Surgery 2018;164(03):489-493

12 Kelly JM, Rizoli S, Veigas P, Hollands S, Min A. Using rotational thromboelastometry clot firmness at 5 minutes $\left(\right.$ ROTEM $^{\circledR}$ EXTEM A5) to predict massive transfusion and in-hospital mortality in trauma: a retrospective analysis of 1146 patients. Anaesthesia 2018;73(09):1103-1109

13 Laursen TH, Meyer MAS, Meyer ASP, et al. Thrombelastography early amplitudes in bleeding and coagulopathic trauma patients: results from a multicenter study. J Trauma Acute Care Surg 2018; 84(02):334-341

14 Scala E, Coutaz C, Gomez F, Alberio L, Marcucci C. Comparison of ROTEM sigma to standard laboratory tests and development of an algorithm for the management of coagulopathic bleeding in a tertiary center. J Cardiothorac Vasc Anesth 2020;34(03):640-649

15 Kozek-Langenecker SA, Ahmed AB, Afshari A, et al. Management of severe perioperative bleeding: guidelines from the European Society of Anaesthesiology: first update 2016. Eur J Anaesthesiol 2017;34(06):332-395

16 Ziegler B, Voelckel W, Zipperle J, Grottke O, Schöchl H. Comparison between the new fully automated viscoelastic coagulation analysers TEG 6s and ROTEM Sigma in trauma patients: a prospective observational study. Eur J Anaesthesiol 2019;36(11): 834-842

17 Bouzat P, Guerin R, Boussat B, et al. Diagnostic performance of thromboelastometry in trauma-induced coagulopathy: a comparison between two level I trauma centres using two different devices. Eur J Trauma Emerg Surg 2019 (e-pub ahead of print) . Doi: 10.1007/s00068-019-01165-7

18 Dötsch TM, Dirkmann D, Bezinover D, et al. Assessment of standard laboratory tests and rotational thromboelastometry for the prediction of postoperative bleeding in liver transplantation. Br J Anaesth 2017;119(03):402-410

19 Haas T, Fries D, Tanaka KA, Asmis L, Curry NS, Schöchl H. Usefulness of standard plasma coagulation tests in the management of perioperative coagulopathic bleeding: is there any evidence? Br J Anaesth 2015;114(02):217-224

20 Stettler GR, Moore EE, Nunns GR, et al. Rotational thromboelastometry thresholds for patients at risk for massive transfusion. J Surg Res 2018;228:154-159

21 Stettler GR, Sumislawski JJ, Moore EE, et al. Citrated kaolin thrombelastography (TEG) thresholds for goal-directed therapy in injured patients receiving massive transfusion. J Trauma Acute Care Surg 2018;85(04):734-740

22 Santos AS, Oliveira AJF, Barbosa MCL, Nogueira JLDS. Viscoelastic haemostatic assays in the perioperative period of surgical procedures: systematic review and meta-analysis. J Clin Anesth 2020;64:109809 
23 Wikkelsø A, Wetterslev J, Møller AM, Afshari A. Thromboelastography (TEG) or thromboelastometry (ROTEM) to monitor haemostatic treatment versus usual care in adults or children with bleeding. Cochrane Database Syst Rev 2016;(08):CD007871

24 Li C, Zhao Q, Yang K, Jiang L, Yu J. Thromboelastography or rotational thromboelastometry for bleeding management in adults undergoing cardiac surgery: a systematic review with meta-analysis and trial sequential analysis. J Thorac Dis 2019; 11(04):1170-1181

25 Anderson L, Quasim I, Soutar R, Steven M, Macfie A, Korte W. An audit of red cell and blood product use after the institution of thromboelastometry in a cardiac intensive care unit. Transfus Med 2006;16(01):31-39

26 Görlinger K, Dirkmann D, Hanke AA, et al. First-line therapy with coagulation factor concentrates combined with point-of-care coagulation testing is associated with decreased allogeneic blood transfusion in cardiovascular surgery: a retrospective, singlecenter cohort study. Anesthesiology 2011;115(06):1179-1191

27 Spiess BD, Gillies BS, Chandler W, Verrier E. Changes in transfusion therapy and reexploration rate after institution of a blood management program in cardiac surgical patients. J Cardiothorac Vasc Anesth 1995;9(02):168-173

28 Karkouti K, Callum J, Wijeysundera DN, et al; TACS Investigators. Point-of-care hemostatic testing in cardiac surgery: a steppedwedge clustered randomized controlled trial. Circulation 2016; 134(16):1152-1162

29 Prat NJ, Meyer AD, Ingalls NK, Trichereau J, DuBose JJ, Cap AP. Rotational thromboelastometry significantly optimizes transfusion practices for damage control resuscitation in combat casualties. J Trauma Acute Care Surg 2017;83(03):373-380

30 Unruh M, Reyes J, Helmer SD, Haan JM. An evaluation of blood product utilization rates with massive transfusion protocol: before and after thromboelastography (TEG) use in trauma. Am J Surg 2019;218(06):1175-1180

31 Stein P, Kaserer A, Sprengel K, et al. Change of transfusion and treatment paradigm in major trauma patients. Anaesthesia 2017; 72(11):1317-1326

32 Gonzalez E, Moore EE, Moore HB, et al. Goal-directed hemostatic resuscitation of trauma-induced coagulopathy: a pragmatic randomized clinical trial comparing a viscoelastic assay to conventional coagulation assays. Ann Surg 2016;263(06):10511059

33 Snegovskikh D, Souza D, Walton Z, et al. Point-of-care viscoelastic testing improves the outcome of pregnancies complicated by severe postpartum hemorrhage. J Clin Anesth 2018; 44:50-56

34 McNamara H, Kenyon C, Smith R, Mallaiah S, Barclay P. Four years' experience of a ROTEM $^{\circledR}$-guided algorithm for treatment of coagulopathy in obstetric haemorrhage. Anaesthesia 2019;74 (08):984-991

35 Gratz J, Güting H, Thorn S, et al. Protocolised thromboelastometricguided haemostatic management in patients with traumatic brain injury: a pilot study. Anaesthesia 2019;74(07):883-890
36 Shander A, Görlinger K. Blindspots and limitations in viscoelastic testing in pregnancy. Int J Obstet Anesth 2019;38:4-9

37 Rick ME, Walsh CE, Key NS. Congenital bleeding disorders. Hematology (Am Soc Hematol Educ Program) 2003;1:559-574

38 Casonato A, Galletta E, Sarolo L, Daidone V. Type 2N von Willebrand disease: characterization and diagnostic difficulties. Haemophilia 2018;24(01):134-140

39 Chen A, Teruya J. Global hemostasis testing thromboelastography: old technology, new applications. Clin Lab Med 2009;29(02): 391-407

40 Neal MD, Moore EE, Walsh M, et al. A comparison between the TEG 6s and TEG 5000 analyzers to assess coagulation in trauma patients. J Trauma Acute Care Surg 2020;88(02):279-285

41 Forman KR, Wong E, Gallagher M, McCarter R, Luban NL, Massaro AN. Effect of temperature on thromboelastography and implications for clinical use in newborns undergoing therapeutic hypothermia. Pediatr Res 2014;75(05):663-669

42 Kander T, Brokopp J, Friberg H, Schött U. Wide temperature range testing with ROTEM coagulation analyses. Ther Hypothermia Temp Manag 2014;4(03):125-130

43 Bulanov AIu, Iatskov KV, Shulutko EM, Glukhova TE, Andreǐchenko SA. Endogenous heparin-like syndrome: analysis of clinical observations [in Russian]. Anesteziol Reanimatol 2012;(03):51-54

44 Senzolo M, Cholongitas E, Thalheimer U, et al. Heparin-like effect in liver disease and liver transplantation. Clin Liver Dis 2009;13 (01):43-53

45 Ranucci M, Baryshnikova E, Isgrò G, et al. Heparin-like effect in postcardiotomy extracorporeal membrane oxygenation patients. Crit Care 2014;18(05):504

46 Ichikawa J, Kodaka M, Nishiyama K, Hirasaki Y, Ozaki M, Komori M. Reappearance of circulating heparin in whole blood heparin concentration-based management does not correlate with postoperative bleeding after cardiac surgery. J Cardiothorac Vasc Anesth 2014;28(04):1003-1007

47 Sørensen B, Johansen P, Christiansen K, Woelke M, Ingerslev J. Whole blood coagulation thrombelastographic profiles employing minimal tissue factor activation. J Thromb Haemost 2003;1 (03):551-558

48 Rivard GE, Brummel-Ziedins KE, Mann KG, Fan L, Hofer A, Cohen E. Evaluation of the profile of thrombin generation during the process of whole blood clotting as assessed by thrombelastography. J Thromb Haemost 2005;3(09):2039-2043

49 Corey FS, Walker WF. Sonic estimation of elasticity via resonance: a new method of assessing hemostasis. Ann Biomed Eng 2016;44 (05):1405-1424

50 Tripathi MM, Hajjarian Z, Van Cott EM, Nadkarni SK. Assessing blood coagulation status with laser speckle rheology. Biomed Opt Express 2014;5(03):817-831

51 Hartmann J, Murphy M, Dias JD. Viscoelastic hemostatic assays: moving from the laboratory to the site of care-a review of established and emerging technologies. Diagnostics (Basel) 2020;10(02): E118 